\title{
Cortical Involvement in the Induction, But Not Expression, of Thalamic Plasticity
}

\author{
Jayson L. Parker and Jonathan O. Dostrovsky \\ University of Toronto, Department of Physiology, Toronto, Ontario M5S 1A8, Canada
}

The present study examined the role of the somatosensory cortex in the plasticity of thalamic sensory maps. Thalamic plasticity was induced by the disruption of hindlimb input by unilateral destruction of nucleus gracilis. Unilateral somatosensory cortex lesions were performed either on the same day as or a week after the removal of hindlimb input. Multiple electrode penetrations enabled us to measure the volume of somatosensory thalamus devoted to hindlimb, forepaw, and shoulder body regions.

Cortical lesions alone did not change the volume of the shoulder, forepaw, or hindlimb representations in the thalamus relative to controls. However, these lesions blocked the in-

It has been demonstrated for some time that removal of sensory input in the adult results in the expansion of adjacently represented regions with intact sensory inputs into the region that has lost its normal input (Wall and Egger, 1971; Basbaum and Wall, 1976; Dostrovsky et al., 1976; Devor and Wall, 1978; Merzenich et al., 1978; Kalaska and Pomeranz, 1979; Florence and Kaas, 1995). Recently we showed that the removal of hindlimb input to thalamus by nucleus gracilis lesions in adult rats precipitated the expansion of the shoulder representation in the rostral ventroposterolateral nucleus (VPL) of the thalamus as early as 1 week after the lesions and was still observed 4 weeks later (Parker et al., 1998). This region was described in detail and was referred to as a "focal zone" of plasticity. In our paradigm, multiple electrode penetrations allow us to measure the volume of somatosensory thalamus devoted to a given body region. An unusual aspect of the plasticity in this system is that after the disruption of hindlimb input, the shoulder representation expands to overlap the adjacent forelimb representation, a form of somatotopic reorganization that had not been observed before (Fig. 1; Parker et al., 1998).

In the present study, the role of the somatosensory cortex in both the induction and expression of thalamic plasticity induced by partial removal of sensory inputs from the hindlimb by nucleus gracilis lesions is investigated. The induction phase refers to the period when it is thought that reorganization is taking place, whereas expression refers to the period when the changes have already taken place and are maintained. The distinction between these two periods can be demonstrated if a manipulation disrupts the reorganization at an early time period (e.g., the same day) but

\footnotetext{
Received Feb. 17, 1999; revised July 1, 1999; accepted July 16, 1999.

This work was supported in part by National Institutes of Health Grant NS36824. Correspondence should be addressed to J. O. Dostrovsky, Department of Physiology, University of Toronto, Medical Sciences Building Room 3305, Toronto, Ontario, Canada M5S 1A8.

Copyright (C) 1999 Society for Neuroscience $\quad 0270-6474 / 99 / 198623-07 \$ 05.00 / 0$
}

crease in shoulder representation resulting from the nucleus gracilis lesion. In contrast, if thalamic reorganization caused by removal of hindlimb input was allowed to occur, subsequent somatosensory cortex lesions 1 week later did not prevent reorganization. Thus, an intact somatosensory cortex is necessary for the occurrence of sensory map reorganization at the thalamic level (induction) in response to nucleus gracilis lesions, but not for the maintenance of such changes once they are present (expression).

Key words: VPL; thalamus; nucleus gracilis; somatosensory cortex; plasticity; induction; expression; deafferentation

fails to have any effects if performed in a later time period (e.g., 1 week later).

In the case of thalamic plasticity, if the cortex is necessary for the initiation of reorganization of somatotopy in VPL after the removal of hindlimb input, this is referred to as induction. If the cortex is necessary for maintaining the reorganized somatotopy in the VPL 1 week after the removal of hindlimb input, this is referred to as expression. The effects of cortical lesions alone were also examined in this paradigm.

The cortical representation of the shoulder was chosen for aspiration because it is this body region that undergoes increased representation in the thalamus after nucleus gracilis lesions (Parker et al., 1998).

\section{MATERIALS AND METHODS}

Subjects and groups. Thirty-three male Wistar rats with weights ranging from 250 to $350 \mathrm{gm}$ were used. Five treatment groups of adult rats were used: sham nucleus gracilis and sham cortical lesions (Sham; $n=6$ ); nucleus gracilis lesions ( $\mathrm{Gr} ; n=7$ ); somatosensory cortex lesions (CTX; $n=6$ ); nucleus gracilis and immediate somatosensory cortex lesions ( $\mathrm{Gr}$ \& CTX; $n=8$ ); and nucleus gracilis lesions followed 1 week later by somatosensory cortex lesions (Expression; $n=6$ ). All experimental protocols were approved by the University of Toronto Animal Care Committee and conducted in accordance with Canadian Council on Animal Care guidelines.

Nucleus gracilis and somatosensory cortex lesions. Rats were anesthetized with ketamine hydrochloride $(200 \mathrm{mg} / \mathrm{kg}$, i.p.) and xylazine (50 $\mathrm{mg} / \mathrm{kg}$, i.p.). The rat's head was tilted forward in the stereotaxic frame, and the obex was exposed surgically. Under a dissecting microscope, lesions were made of the gracile nucleus with jeweler's forceps. The nucleus was completely macerated with the forceps along its length. Pilot work had established by multiunit recordings and subsequent histological analysis that the boundary between nucleus cuneatus and gracilis was easily predicted on the basis of surface landmarks. Cortical lesions were small and involved primarily the forelimb representation $\left(\sim 5 \mathrm{~mm}^{2}\right)$. This region was identified on the basis of the locations of sites where forelimb responses were elicited during microelectrode mapping of the cortex (a grid of tracks separated by $400 \mu \mathrm{m}$ ). The lesions were made by aspiration using a Pasteur pipette mounted on a stereotaxic arm that could be moved in stereotaxic coordinates. The depth of lesions was visually 


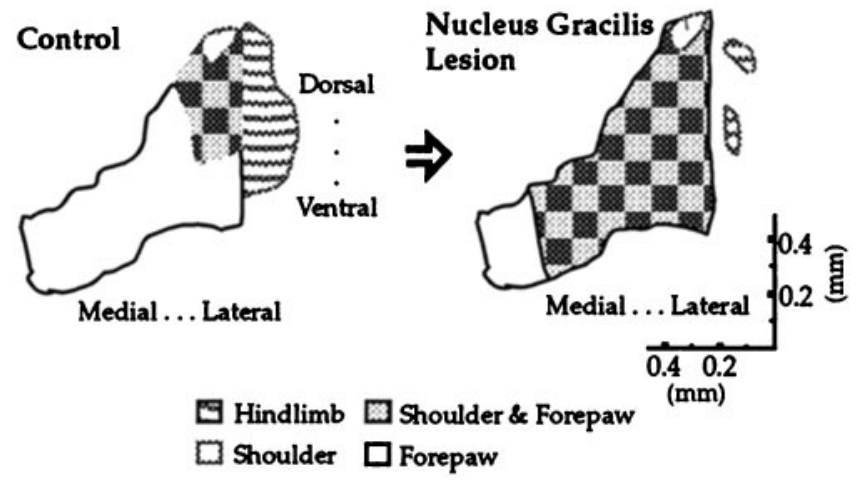

Figure 1. A summary of previous results obtained in this system: the reorganization of the shoulder representation after partial deafferentation of the hindlimb by nucleus gracilis lesions (Parker et al., 1998). The schematic depicts a coronal view of the VPL nucleus. All reorganization occurred within a focal zone located toward the rostral pole of the VPL. Representative data from which this figure was derived are shown in Figure 3.

guided, so as to avoid damage to the underlying hippocampus. All animals received the sham version of the surgical protocol if they were not to be lesioned. These sham controls had both a craniotomy and surgical exposure of the obex and removal of the dura at both sites.

Surgery. Animals were water-deprived overnight before mapping of the thalamus. Five minutes before anesthesia commenced, atropine sulfate $(300 \mathrm{mg} / \mathrm{kg}$, s.c.) was injected. Ketamine hydrochloride $(200 \mathrm{mg} / \mathrm{kg}$, i.p. $)$ and xylazine $(50 \mathrm{mg} / \mathrm{kg}$, i.p.) were administered to anesthetize the animal. Maintenance doses of ketamine hydrochloride and xylazine were given every hour at one quarter the size of the animal's initial dose. Anesthetic level was monitored by ensuring that there was no response to tail pinch, whereas gentle touch to the eye yielded a weak corneal reflex. In the event of a response to tail pinch or a strong corneal reflex, a maintenance dose of anesthetic was administered. Atropine sulfate was administered every $2 \mathrm{hr}(300 \mathrm{mg} / \mathrm{kg}$, s.c.). Body temperature was monitored with a rectal thermometer and kept at $\sim 37^{\circ} \mathrm{C}$ by a thermostatically controlled heating pad underneath the animal. A hole in the trachea was cut, and periodic cleaning with paper tissue followed for at least $20 \mathrm{~min}$ until secretions stopped accumulating. A polyethylene tube was then inserted into the trachea. A unilateral craniotomy contralateral to the side of the gracile lesion was made, extending to all suture lines and the rhinal fissure. The dura was subsequently removed with forceps. Throughout the experiment, the exposed cortex $\left(\sim 1.5 \mathrm{~cm}^{2}\right)$ was covered with mineral oil.

Recordings. Multiunit recordings were conducted with glass-coated tungsten microelectrodes that had an exposed tip length of $35 \mu \mathrm{m}$ and an impedance between 2.5 and $3.0 \mathrm{M} \Omega$ at $1000 \mathrm{~Hz}$. Low-pass and high-pass filters with cutoff frequencies of 6000 and $300 \mathrm{~Hz}$, respectively, were used. The amplifiers, filters, and oscilloscope had the same settings across recording sessions for every animal. The initial electrode penetrations of each session were performed at anteroposterior, $-3.5 \mathrm{~mm}$ and mediolateral, $\pm 3.3 \mathrm{~mm}$ corresponding to the center of VPL relative to bregma (Paxinos and Watson, 1997). The electrode was inserted $4.5 \mathrm{~mm}$ below the cortical surface. Normally, two or three penetrations were required to locate sites responsive to stimulation of the forelimb or hindlimb (and subsequently confirmed histologically). Mapping was then performed by making successive penetrations in a two-dimensional grid of electrode penetrations in the rostrocaudal and mediolateral planes in $0.2 \mathrm{~mm}$ increments, with successive recordings in the dorsoventral axis at the same increments. To reduce the mapping time, a restricted number of body regions was examined. For each recording site (Figs. 2, 3), we tested whether the units discharged to application of brush stimuli applied to either the forepaw (from elbow to paw), shoulder (from elbow to upper extremity of the arm), or hindlimb (thigh or foot). The term forelimb refers to forepaw and shoulder body regions. If a site failed to respond to stimulation of one of these body regions, it was classified as "blank". If a site responded to more than one body region, each one was noted. At the end of the mapping session, two electrolytic lesions were placed (one caudal, one anterior, at the same depth), and the coordinates recorded. The rat was then killed by anesthetic overdose.
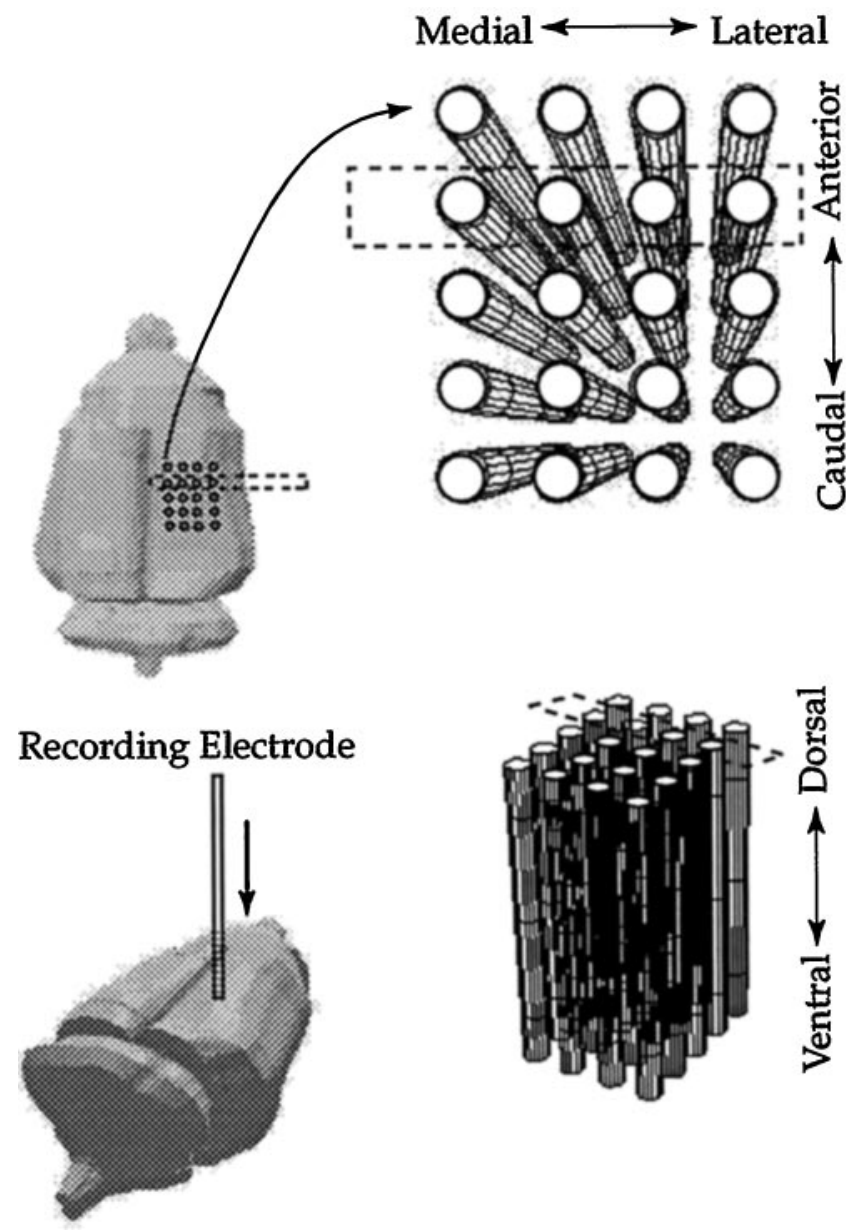

Figure 2. An illustration of the grid of electrode recordings used and how these data correspond to different axes in the intact rat brain. Most of the plasticity in this system is located in a confined area, previously described as a focal zone located toward the rostral pole of the VPL (Parker et al., 1998). For clarity of presentation, the grid shown has fewer penetrations than are normally used.

Sensory maps. The forepaw sensory map included any site responsive to forepaw stimulation, regardless of whether the same site was also responsive to other body regions. Likewise, the shoulder sensory map included all sites responsive to shoulder stimulation, ignoring the fact that some sites may have been responsive also to other body regions. The zone of overlap between shoulder and forepaw consisted of all those sites responsive to both shoulder and forepaw stimulation, regardless of responsiveness to the stimulation of other body regions.

Mapping rules. The following protocol was followed in order to map the forepaw representation. This protocol produced consistent sensorymap data across pilot animals with respect to volume and shape. (1) Recording commenced in a new track $0.6 \mathrm{~mm}$ above the most dorsal positively identified site obtained in any adjacent electrode track; (2) each penetration continued until it was $0.6 \mathrm{~mm}$ below the most ventral positively identified site in that track, and if no positively identified sites were encountered within an electrode track, the penetration continued until it was $0.6 \mathrm{~mm}$ below the lowest positive site in any adjacent electrode track; (3) successive penetrations medially and laterally in the mediolateral axis were terminated after two contiguous blank penetrations were encountered (sample data in Fig. 3); (4) an additional row of electrode penetrations was performed $0.2 \mathrm{~mm}$ rostral and caudal to a completed series of tracks in a given coronal plane if the previous row of electrode penetrations revealed a site responsive to somatic stimulation of the forelimb, shoulder, or hindpaw. Such rules generated an aggregate map in the VPL that represented all three body regions examined. Such maps required $80-100$ penetrations per animal and contained $\sim 400$ positively identified sites per animal. On average, from tracheotomy to 


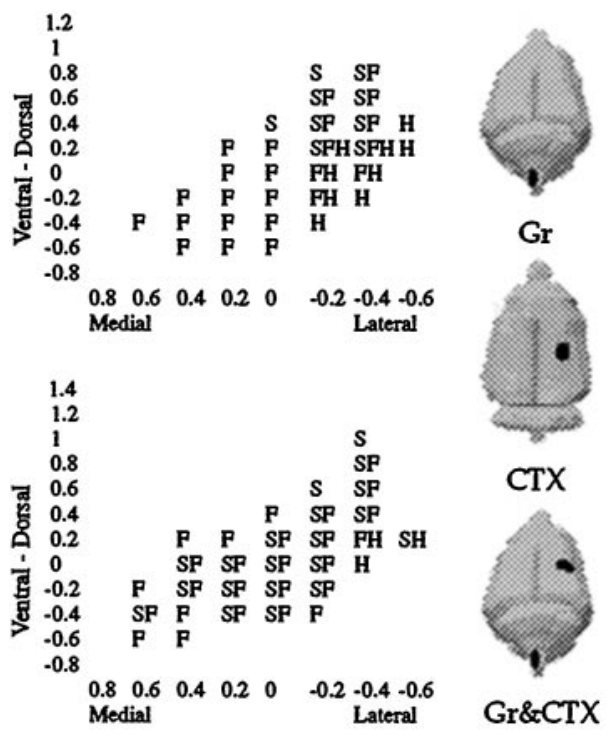

Figure 3. A coronal view of the VPL depicting recording sites responsive to tactile stimulation of the hindlimb, shoulder, and/or forepaw, corresponding to the row of electrode penetrations enclosed by a dashed rectangle in Figure 2. Typical data from Sham and Gr animals are shown. Three figurines illustrate three of the principal combinations of lesions used in this study and their ipsilateral and contralateral relationships.

perfusion, the procedure required $12-14 \mathrm{hr}$ per animal. For each rat thalamus, a series of spreadsheets described in stereotaxic coordinates the three-dimensional pattern of positively identified recording sites.

Lesion history. The Expression group did not receive cortical lesions until 1 week after the nucleus gracilis lesion was performed. This allowed thalamic reorganization induced by the gracile lesion to occur before the cortical lesion was made. The Expression group was mapped 1 week after the cortical lesion and thus 2 weeks after the nucleus gracilis lesion. All other groups were mapped 1 week after the nucleus gracilis lesion. It is known from a previous study (Parker et al., 1998) that volumetric increases in the shoulder representation are complete 1 week after nucleus gracilis lesions and have the same magnitude when examined 1 month later. The placement of these lesions is shown in a simple schematic in Figure 3 for the CTX, Gr, and Gr \& CTX groups.

Volumetric and spatial analysis. Sensory maps were reconstructed and visualized in three dimensions with the Interactive Data Language program (IDL, Boulder, CO) on a Sun Ultra model 170 Sparcstation (Sun Microsystems, Mountain View, CA) from the data recorded on spreadsheets. IDL was used to read in the spreadsheets for a single rat brain and visually reconstruct the spatial distribution of positively identified recording sites for a given body region in three dimensions and manipulate these images on the computer screen. The volume of each sensory map was calculated from the number of recording sites obtained per body region multiplied by the volume element $\left(0.008 \mathrm{~mm}^{3}\right)$ of the grid. Volumetric analysis allowed us to ascertain which body representations underwent expansion. Spatial analysis was then conducted on these sensory maps to determine the topological features mediating the volumetric changes. Volumetric analysis gives us the total volume of a sensory map, whereas spatial analysis gives us cross-sectional areas of the sensory map at regular intervals, to quantify its characteristic shape (e.g., the map is sliced in IDL). A series of coronal areas of three-dimensional reconstructions of the shoulder sensory map were compared between groups. Equivalent coronal slices of the shoulder sensory map across animals were identified by their distance from a neuroanatomical landmark. Successive cross-sectional areas of the maps in the coronal, sagittal, and horizontal planes were compared between groups. Equivalent slices of a given sensory map across animals were identified by virtue of their distance from the midpoint of the length of the ventrobasal complex (VB) along the anteroposterior axis.

Statistics. If the requirement of homogeneity of variance was unsatisfied for a given ANOVA, a correction was performed to the number of degrees of freedom with Huynh-Feldt Epsilon, making the required level of significance more stringent. If normality was violated, a nonparametric

\section{Dimensional Reconstructions of the Shoulder Sensory Map}
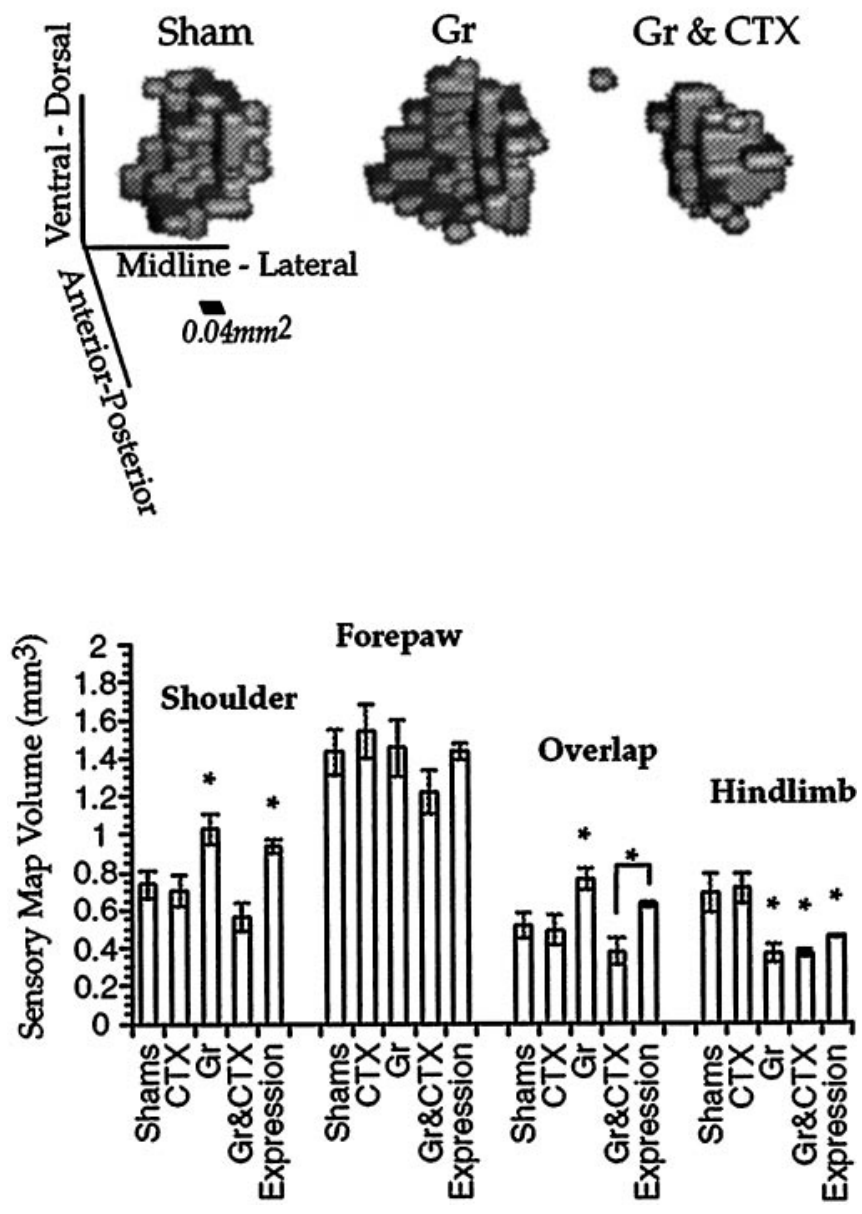

Figure 4. Representative three-dimensional reconstructions of the shoulder sensory map. Relative to Sham, CTX, and Gr \& CTX, both Gr and Expression shoulder sensory map volumes are significantly elevated. The results are similar for the shoulder sensory map and the region of overlap between shoulder and forepaw sensory maps. However, the elevation in the Expression group is not statistically significant for the sensory map representing this overlap. Error bars indicate SEM.

test was used. If the results of the nonparametric test were the same as an ANOVA, the ANOVA was reported. All statistics were performed with SPSS (SPSS Inc., version 6.1 for Macintosh, Chicago, IL). Post hoc unpaired $t$ test (two-tailed) comparisons that followed ANOVA models are reported as being less than or greater than a 0.05 significance level.

Histology. Animals were perfused transcardially with saline followed by $4 \%$ paraformaldehyde. Brains were removed and placed in a $30 \%$ sucrose solution of $4 \%$ paraformaldehyde. Half the rat brains were sectioned in the horizontal plane with a slice thickness of $0.1 \mathrm{~mm}$. This plane of sectioning allowed us to discern the entire pattern of electrode tracks in VPL. In the other half of the rats, the brains were sectioned coronally at $40 \mu \mathrm{m}$ to assess cortical lesions and to confirm that damage did not extend below the depth of the cortex. Dorsal column nuclei were sectioned coronally with a slice thickness of $40 \mu \mathrm{m}$. All tissue was Nissl-stained.

\section{RESULTS}

\section{Volumetric analysis}

A representative three-dimensional reconstruction of the shoulder sensory map in the thalamus for each of three treatment conditions is shown in Figure 4. Each voxel of the display repre- 
sents a recording site that was responsive to shoulder stimulation. Sensory maps are viewed from a caudal perspective. Visual inspection suggests that the volumetric expansion in the shoulder sensory map in the Gr group relative to the Sham group is blocked by the cortical lesions in the Gr \& CTX group. The Gr \& CTX group sensory map actually appears smaller than the Sham group sensory map, but this trend was not supported in the quantitative analysis below.

Volumetric analysis of the shoulder sensory map supported many of the trends observable in the three-dimensional reconstructions (Fig. 4). An aggregate comparison among all groups for all these four body regions in a multivariate statistical model revealed a significant main effect of group [Pillias $(3.67,112)=$ $1.38 ; p<0.001]$ with a power of 1.0 and an overall effect size of 0.344. Univariate ANOVAs are presented for each sensory map in turn. The power of each ANOVA reported is $>0.95$, with the exception of the forepaw analysis, which had a power of 0.31 . Simultaneous lesions of the cortex and nucleus gracilis blocked thalamic plasticity of the shoulder sensory map normally observed with nucleus gracilis lesions alone (one-way ANOVA; $F_{(4,28)}=$ 7.27; $p<0.001$ ). Relative to the Sham condition, the CTX group was not significantly different (post hoc $t$ test). In the Gr group there was a clear volumetric increase of the shoulder sensory map relative to the Sham group (post hoc $t$ test), consistent with what has been reported previously (Parker et al., 1998). The volume of the shoulder sensory map in the Gr \& CTX group was no different from that in the sham controls and was significantly depressed relative to that in the Gr group (post hoc $t$ tests). Furthermore, the volume of the shoulder sensory map in the Expression group was similar to that in the Gr group and was significantly elevated relative to those in the Sham, CTX, and Gr \& CTX groups (post hoc $t$ tests).

In contrast, the forepaw sensory map did not change in volume in any of the treatment conditions (Fig. 4). A one-way ANOVA did not reveal any treatment effect $\left(F_{(4,28)}=1.85 ; p=0.364\right)$. With the possible exception of the Gr \& CTX group, the volume of this representation appears consistent when compared across treatment groups.

The pattern of results in the volume of sensory map overlap between shoulder and forepaw is similar to that obtained with the shoulder sensory map alone, albeit with some differences (Fig. 4). A one-way ANOVA revealed an overall treatment effect $\left(F_{(4,28)}\right.$ $=6.4 ; p<0.001)$. The volume of overlap between the forepaw and shoulder sensory maps expanded in a robust manner after nucleus gracilis lesions relative to Sham and CTX groups (post hoc $t$ tests). This expansion was blocked by cortical lesions and was not significantly different from CTX and Sham groups (post hoc $t$ tests). The Expression group was significantly elevated relative to the Gr \& CTX group but not the CTX or the Sham group. Furthermore, the volume of overlap in the expression group was significantly lower than the volume of that in the $\mathrm{Gr}$ group (post hoc $t$ tests).

Residual hindlimb input to the VPL was analyzed (Fig. 4), and a main effect of nucleus gracilis lesions was found (one-way ANOVA; $\left.F_{(4,28)}=16.1 ; p<0.0001\right)$. The hindlimb functional maps in all three groups receiving nucleus gracilis lesions ( $\mathrm{Gr}, \mathrm{Gr}$ \& CTX, and Expression) were significantly depressed relative to controls (Sham and CTX; post hoc $t$ tests). Approximately $50 \%$ of the hindlimb functional map volume appeared to be lost in the group that received nucleus gracilis lesions. Relative to the $\mathrm{Gr} \&$ CTX group, the expression group was significantly elevated, but not with respect to the Gr group (post hoc $t$ tests).

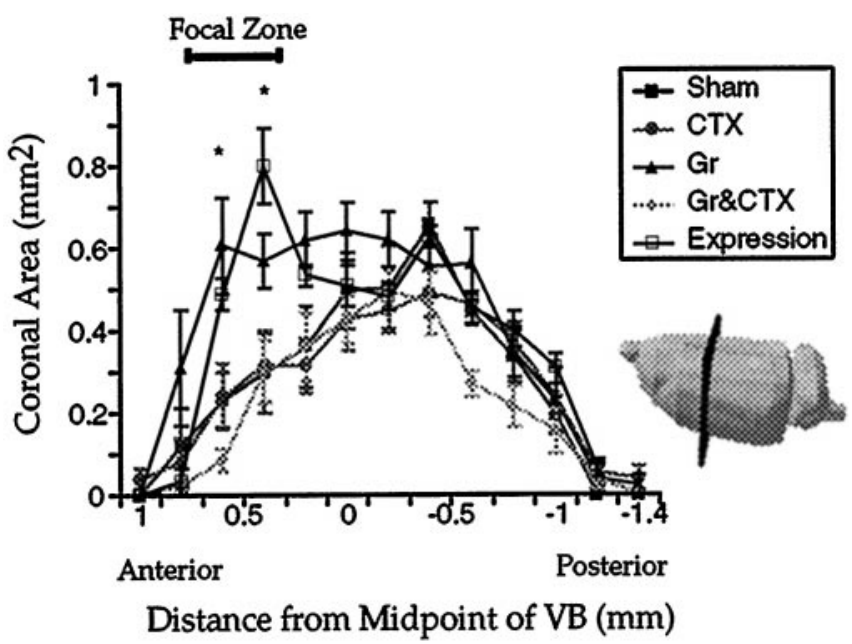

Figure 5. Areas of successive coronal sections of the shoulder sensory map as a function of the anteroposterior distance. Coronal slicing of three-dimensional shoulder sensory maps were performed on a computer. Each slice was identified by its distance from the midpoint of the VB along the anteroposterior plane. The figure shows the orientation of slicing of the shoulder sensory map with respect to intact rat brain. Error bars indicate SEM.

\section{Region of the volumetric increase}

Finally, the area of the volumetric change in the thalamic shoulder sensory map was examined by sectioning these functional maps in the coronal plane (Fig. 5; two-way ANOVA; effect of group, $F_{(4,28)}=6.75, p<0.002$; slice by group interaction, $\left.F_{(48,248.57)}=35.51, p<0.001\right)$. Power was $>0.95$, and the effect of size was 0.50 . As expected, all the expansion was localized to a zone located toward the rostral pole of the shoulder sensory map. Groups that had a somatosensory cortical lesion (CTX; Gr \& CTX) showed the same successive cross-sectional areas as the Sham group at all anteroposterior levels of the VPL. In contrast, the Expression group had the same series of cross-sectional areas as the Gr group, as it was significantly elevated relative to all other groups in the focal zone (post hoc $t$ tests).

\section{Histology}

Figure 6 shows a typical cortical lesion. As can be seen in this photomicrograph, damage was confined to the cortex; both the VPL and the overlaying hippocampus were undamaged. In a coronal view, some electrode tracks are discernible from mapping the VPL. The site of damage is well removed from the SII cortical representation, which is found on the lateral aspect of the rat brain (Chapin and Chia-Sheng, 1990). Figure 7 presents a reconstruction of electrode recording sites in the VPL based on horizontal sections through the thalamus in a sham control and gracile nucleus lesioned animal. The focal zone of plasticity is indicated in the lesioned animal, and the predicted corresponding location is indicated in the control animal. Finally, damage to nucleus gracilis is also shown in a Nissl-stained transverse section (Fig. $8 A, B)$. The lesion completely destroyed nucleus gracilis, which was observed along the length of the nucleus, while sparing nucleus cuneatus.

\section{DISCUSSION}

The data presented here provide strong support for a permissive role of the somatosensory cortex in the induction, but not expression, of thalamic plasticity. This effect was illustrated by the 


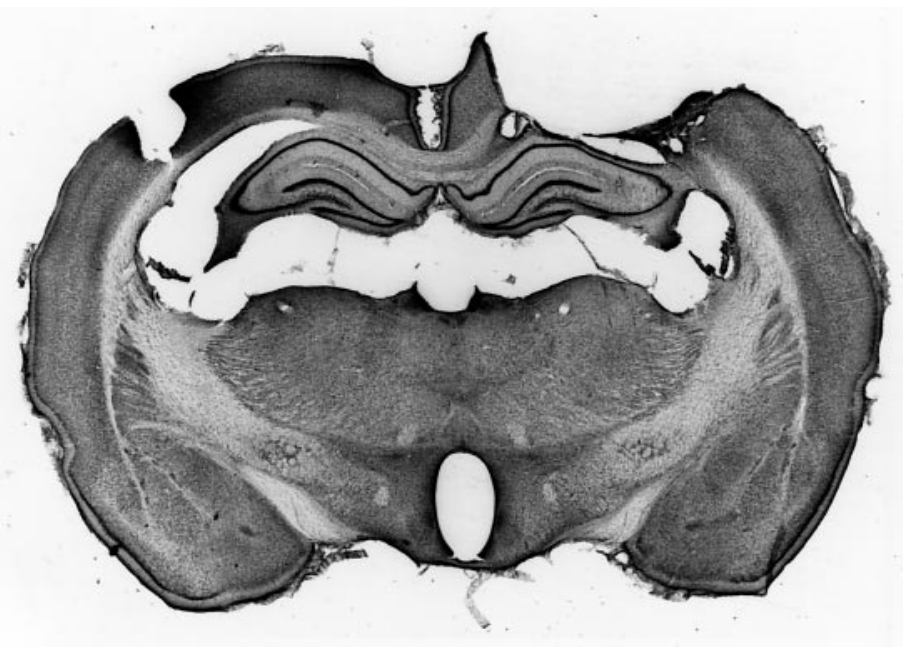

Figure 6. A coronal view of the cerebral cortex that received an aspirative lesion. The section has been Nissl-stained. The zone of damage was guided by electrode mapping of neurons responsive to contralateral forelimb stimulation with brush stimuli. The lateral aspect of the lesion stopped at the rhinal fissure. The lesion spared the underlying hippocampus and thalamus.

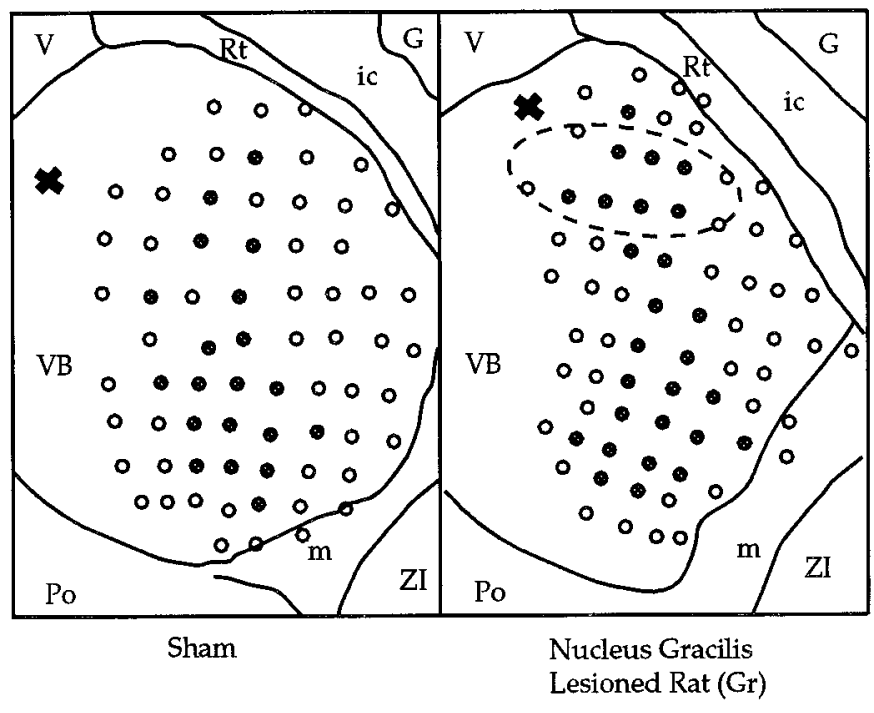

Figure 7. Reconstructions of thalamic electrode penetrations for a Sham and $\mathrm{Gr}$ animal are presented, viewed dorsally. Tissue was sectioned in the horizontal plane and Nissl-stained. An electrolytic lesion $(X)$ at the top left of each schematic served as a reference point along the dorsoventral axis. The focal zone of plasticity of the VPL is indicated (ellipse). All electrode tracks that encountered sites responsive to shoulder stimulation are indicated by filled circles. $G$, Globus pallidus; $i c$, internal capsule; $m$, medial lemniscus; $P o$, posterior thalamic nuclear group; $R t$, reticular thalamic nucleus; $V$, third ventricle; $Z I$, zona incerta.

changes in the thalamic shoulder representation induced by nucleus gracilis lesions. Somatosensory cortex lesions prevented the volumetric increase in the thalamic shoulder sensory map when performed on the same day as nucleus gracilis lesions. This finding indicates that an intact somatosensory cortex is necessary to permit the induction of thalamic plasticity after nucleus gracilis lesions. In contrast, if plasticity was allowed to occur at the thalamic level after nucleus gracilis lesions, subsequent somatosensory cortex lesions performed 1 week later had no significant
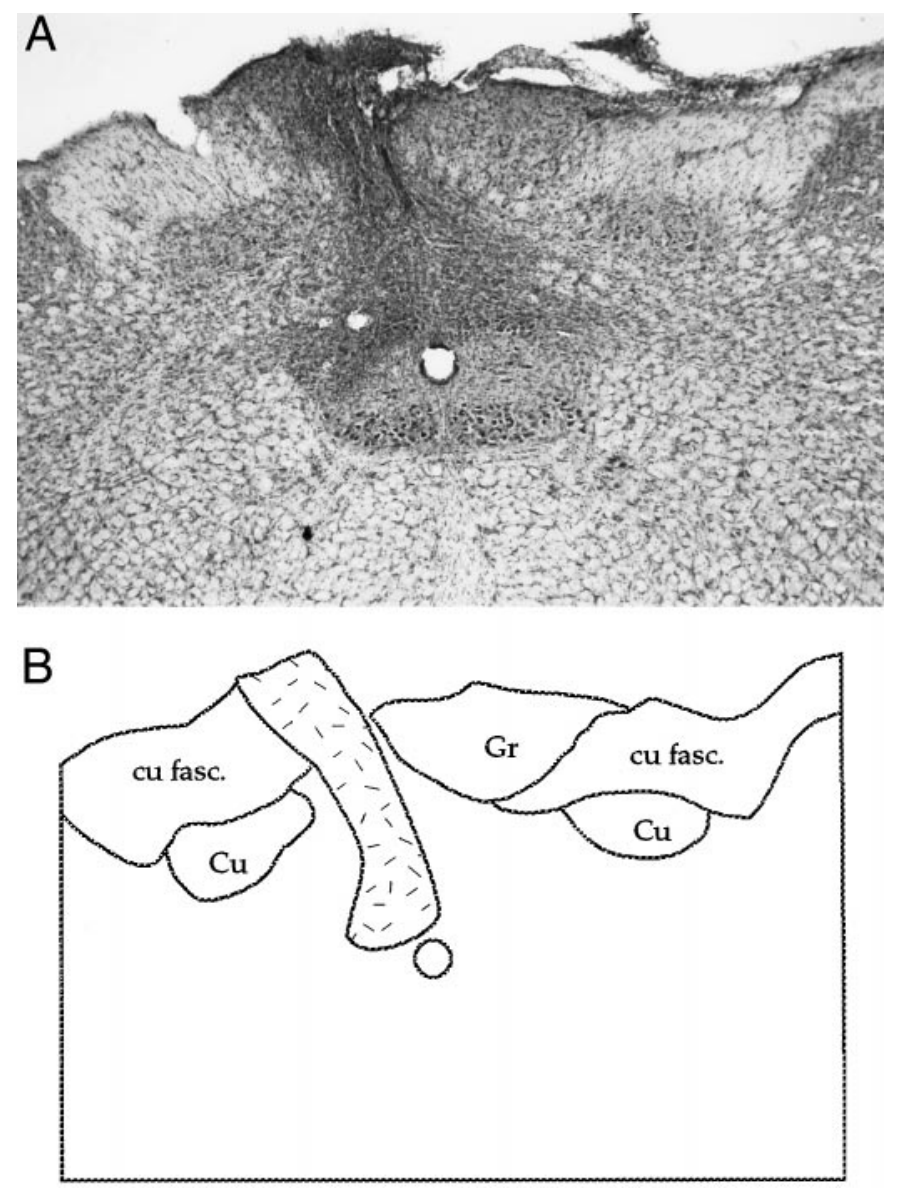

Figure 8. A, Photomicrograph of a nucleus gracilis lesion; $B$, an accompanying schematic: nucleus gracilis $(G r)$, nucleus cuneatus $(\mathrm{Cu})$, and cuneatus fasciculus ( $c$ fasc.). A dense gliotic zone demarcates what remains of the lesioned gracile nucleus (stippled area). Such damage was observed along the length of nucleus gracilis.

effect. This latter finding indicates that the somatosensory cortex is not necessary for the expression of thalamic plasticity.

The topology of the volumetric change of the shoulder sensory map also revealed a great deal of specificity regarding the effect of somatosensory lesions. When somatosensory cortex lesions were performed the same day as nucleus gracilis lesions (Gr \& CTX), these animals had identical successive cross-sectional coronal areas as Sham controls. Thus, somatosensory cortex lesions blocked the volumetric increase of the shoulder representation in the focal zone of the VPL, but did not affect any other segment of the shoulder sensory map. Likewise, the increase in shoulder volume in the Expression group occurred only in the focal zone, as was the finding in the Gr group.

Considerable controversy exists in the literature regarding what effect cortical inactivation has on thalamic neurons (Sherman and Guillery, 1996). Some investigators have reported facilitation of evoked activity after cortical inactivation, whereas others have reported the opposite (Kalil and Chase, 1970; Baker and Malpelli, 1977; Albe-Fessard et al., 1983; Yuan et al., 1985; McClurkin et al., 1994; Sherman and Guillery, 1996). These studies involved acute preparations, in contrast to the chronic time scales permitted by the paradigm employed here. Perhaps the difference in time scales between our study and earlier work accounts for our finding of no effect of somatosensory cortical lesions. Alterna- 
tively, since we are measuring overall sensory maps, the effects of facilitation or inactivation may be averaged out at a population level. Although there may be effects of cortical inactivation on thalamic neurons, with respect to the dependent measure in this study, sensory map volume, there appears to be no effect.

Of more relevance to the present studies, is a recent observation that after both acute and chronic inactivation of the somatosensory cortex with the NMDA antagonist D-2-amino-5phosphonovaleric acid there is expansion of the receptive fields of thalamic VPL neurons (Ergenzinger et al., 1998). Perhaps in our study alterations in cortical processing resulting from loss of hindlimb input give rise to increased receptive field size of neurons in the forelimb/shoulder region that are necessary for the induction of thalamic plasticity. In addition, because ketamine is a glutaminergic antagonist, this report also raises the possibility that the receptive fields of neurons in our study could be larger than those observed in animal studies with a different anesthetic. However, because all groups in this study, including controls, underwent recordings under the same anesthetic, such an effect could not account for the differences observed between the groups.

It is well known that cortical lesions can result in extensive retrograde degeneration of thalamocortical neurons (Bava et al., 1968; Agarwala and Kalil, 1998). However, in our series of experiments no obvious changes in neuronal responses in VPL were observed, and more importantly they did not significantly alter the thalamic sensory map volume in the CTX group relative to Shams. This lack of a significant effect may possibly be caused by the limited extent of the cortical lesion in our study. Finally, it is also possible that cortical lesions resulted in a modest decrease in the density of neurons in the VPL, without altering the relative borders or volumes of the sensory maps.

Some investigators have raised the possibility that the plastic component of vibrissae receptive field structure in the cortex is dependent on intracortical mechanisms (Armstrong-James and Callahan, 1991; Armstrong-James et al., 1991). Cortical lesions suggested that surround-receptive fields (SRFs) were independent of the thalamus and generated through intracortical mechanisms. It was suggested that the long cortical SRF response latency was caused by barrel-to-barrel communication via intracortical routes. Our results suggest a possible alternative interpretation to the lesion study (Armstrong-James et al., 1991) that reported cortical damage to a vibrissa appears to remove it from the SRF of an adjacent cortical barrel. Such a lesion may have instead disrupted corticofugal activation of thalamocortical neurons. Furthermore, corticof ugal activation of thalamocortical neurons may account for the long latency associated with cortical SRFs.

Several investigators have argued that the thalamus and cortex should be considered a loop, rather than the more conventional view that the thalamus passively conveys input to the cortical level (Edelman, 1987; Ergenzinger et al., 1998). The findings of our study support this speculation. Descending input from the cortex appears to play a critical role in plasticity in the thalamus during the induction period. Whereas cortical lesions block thalamic plasticity during induction, it may be that blockade of thalamic plasticity immediately after neural trauma would similarly impair cortical plasticity. Consistent with thalamus and cortex interacting via loops, plasticity in either structure may require that both thalamus (focal zone) and somatosensory cortex are intact. Accordingly, we predict that lesions of the focal zone of the VPL would block cortical plasticity. The control condition would involve lesions placed outside the focal zone in the VPL to ensure that cortical consequences do not reflect a simple disruption in the cutaneous relay properties of the thalamus.

There are several limitations to this study. The lesions of cortex have been crude. It is not known whether functional block of the cortical supragranular, granular, or infragranular layers would be sufficient to prevent thalamic plasticity. Furthermore, it would also be interesting to know how important the size of the cortical lesion is in blocking thalamic plasticity. Finally, there is an issue of paradigm specificity; this result needs to be confirmed with other body regions in different species under different anesthetics, and with different types of plasticity.

The findings reported here raise the possibility that the dorsal column nuclei and spinal cord, like the thalamus, may require an intact cortex to undergo representational plasticity. Perhaps, as Edelman (1987) suggested in his reentrant connectivity model, changes at any given level of the nervous system reflect the engagement of multiple levels of the nervous system.

\section{Conclusion}

In summary, the cortex appears to provide critical input to the thalamus that is necessary to permit it to adapt to changes in sensory input, however, once this has occurred such changes do not require an intact somatosensory cortex to persist.

\section{REFERENCES}

Agarwala S, Kalil RE (1998) Axotomy-induced neuronal death and reactive astrogliosis in the lateral geniculate nucleus following a lesion of the visual cortex in the rat. J Comp Neurol 392:252-263.

Albe-Fessard D, Condes-Lara M, Kesar S, Sanderson P (1983) Tonic cortical controls acting on spontaneous and evoked thalamic activity. In: Somatosensory integration in the thalamus (Macchi G, Rustioni A, Spreafico R, eds), pp 273-285. Amsterdam: Elsevier Science.

Armstrong-James M, Callahan CA (1991) Thalamo-cortical processing of vibrissal information in the rat. II. Spatiotemporal convergence in the thalamic ventroposterior medial nucleus (VPm) and its relevance to generation of receptive fields of S1 cortical "barrel" neurones. J Comp Neurol 303:211-224.

Armstrong-James M, Callahan CA, Friedman MA (1991) Thalamocortical processing of vibrissal information in the rat. I. Intracortical origins of surround but not centre-receptive fields of layer IV neurones in the rat S1 barrel field cortex. J Comp Neurol 303:193-210.

Baker FH, Malpelli JG (1977) Effects of cryogenic blockade of visual cortex on the responses of lateral geniculate neurons in the monkey. Exp Brain Res 29:433-444.

Basbaum AI, Wall PD (1976) Chronic changes in the response of cells in adult cat dorsal horn following partial deafferentation: the appearance of responding cells in a previously non-responsive region. Brain Res 116:181-204.

Bava A, Fadiga E, Manzoni T (1968) Extraleminiscal reactivity and commissural linkages in the VPL nucleus of cats with chronic cortical lesions. Arch Ital Biol 106:204-226.

Chapin JK, Chia-Sheng L (1990) The somatic sensory cortex of the rat. In: The cerebral cortex of the rat (Kolb B, Tees RC, eds), pp 341-380. Cambridge, MA: MIT.

Devor M, Wall PD (1978) Reorganization of spinal cord sensory map after peripheral nerve injury. Nature 275:75-76.

Dostrovsky JO, Millar J, Wall PD (1976) The immediate shift of afferent drive of dorsal column nucleus cells following deafferentation: a comparison of acute and chronic deafferentation in gracile nucleus and spinal cord. Exp Neurol 52:480-495.

Edelman GM (1987) Neural darwinism. New York: Basic Books.

Ergenzinger ER, Glasier MM, Hahm JO, Pons TP (1998) Cortically induced thalamic plasticity in the primate somatosensory system. Nat Neurosci 1:226-229. 
Florence SL, Kaas JH (1995) Large-scale reorganization at multiple levels of the somatosensory pathway follows therapeutic amputation of the hand in monkeys. J Neurosci 15:8083-8095.

Kalaska J, Pomeranz B (1979) Chronic paw denervation causes an agedependent appearance of novel responses from forearm in "paw cortex" of kittens and adult cats. J Neurophysiol 42:618-633.

Kalil RE, Chase R (1970) Corticofugal influences on activity of lateral geniculate nucleus neurons in the cat. Annu Rev Neurosci 33:459-474.

McClurkin JW, Optican LM, Richmond BJ (1994) Cortical feedback increases visual information transmitted by monkey parvocellular lateral geniculate nucleus neurons. Vis Neurosci 11:601-617.

Merzenich MM, Kaas JH, Wall J, Nelson RJ, Sur M, Lin CS (1978) Double representation of the body surface within cytoarchitectonic areas $3 \mathrm{~B}$ and 1 in "S1" in owl monkey (Aotus trivirgatus). J Comp Neurol 181:41-74.

Parker JL, Wood ML, Dostrovsky JO (1980) A focal zone of thalamic plasticity. J Neurosci 18:548-558.

Paxinos G, Watson C (1997) The rat brain in stereotaxic coordinates, Ed 3. San Diego: Academic.

Sherman SM, Guillery RW (1996) Functional organization of thalamocortical relays. J Neurophysiol 76:1367-1395.

Wall PD, Egger MD (1971) Formation of new connexions in adult rat brains after partial deafferentation. Nature 232:542-545.

Yuan B, Morrow TJ, Casey KL (1985) Responsiveness of ventrobasal thalamic neurons after suppression of S1 cortex in the anesthetized rat. J Neurosci 5:2971-2978. 